\title{
Hypoxia-inducible factor-1a contributes to dendritic overgrowth in tuberous sclerosis
}

Longbo Zhang ${ }^{1,2^{*}}$, David M. Feliciano ${ }^{1 * \#}$, Tianxiang Huang ${ }^{1,2}$, Shiliang Zhang $^{1}$, and Angélique Bordey ${ }^{1}$

${ }^{1}$ Departments of Neurosurgery, and Cellular \& Molecular Physiology, Yale University School of Medicine, New Haven, CT 06520-8082 and ${ }^{2}$ Department of Neurosurgery, Xiangya Hospital, Central South University, Changsha, China..

*: First co-authors

\#: present address: Department of Biological Sciences, Clemson University, Clemson, SC 29634-0314.

Address for correspondence: Angélique Bordey, Ph.D.

Department of Neurosurgery

Yale University School of Medicine

333 Cedar Street, FMB 422

New Haven, CT 06520-8082

Phone: 203-737-2515

Fax: 203-737-2159

Email: angelique.bordey@yale.edu 


\begin{abstract}
Expression of hypoxia-inducible factor 1a (HIF1a) is increased under several pathological conditions such as hyperactive mechanistic target of rapamycin complex 1 (mTORC1) in tuberous sclerosis complex (TSC). Hyperactive mTORC1 and the resulting increased dendritic complexity of neurons are shared molecular and cellular alterations in several neurological disorders associated with cognitive disabilities. Despite some evidence that HIF1a contributes to dendritic overgrowth in vitro, it remains unknown whether increased HIF1a in TSC neurons could contribute to their increased dendritic complexity. To address this use in vivo, we generated TSC neurons by deleting Tsc1 in newborn olfactory bulb (OB) neurons of conditional Tscl transgenic mice using neonatal electroporation. In addition to their increased dendritic complexity, $T s c 1^{\text {null }}$ neurons have been reported to display increased Hifla mRNA level and HIF1a transcriptional activity. We found that $T s c 1^{\text {null }}$-dependent dendritic overgrowth was prevented by knocking down HIF1a or expressing a dominant negative HIF1a. In addition, overexpressing HIF1a in wild-type developing neurons resulted in increased dendritic complexity in vivo. These data highlight that an increase in HIF1a levels contributes to abnormal dendritic patterning in developing neurons under normal condition and hyperactive mTORC1 condition as in TSC.
\end{abstract}




\section{Introduction}

Hypoxia-inducible factor 1a (HIF1a) is a basic helix-loop-helix transcription factor and is defined as the $s$ the master transcriptional regulator of cellular and developmental response to hypoxia. Under normoxic conditions, HIF1a undergoes ubiquitination and proteasomal degradation [1]. While in hypoxic conditions, HIF1a accumulates and plays a role in the transcriptional regulation of hypoxia-inducible genes involved in angiogenesis, metabolism, apoptosis, and cellular stress [2;3]. HIF1a is also increased following several pathological conditions, including acute neuroinflammation [4;5] and increased mTORC1 activity in mice and humans independent of hypoxia [6-10]. The function of HIF1a in hyperactive mTORC1 has been shown to increase neuron survival [6]. In this latter study, mTORC1 activity was increased by deleting TSC1, which forms a complex with TSC2 and negatively regulates the activity of the canonical mTORC1 activator, Rheb. Interestingly, in another pathological condition (i.e., lipopolysaccharide (LPS)-modelled inflammation) associated with increased HIF1a levels, HIF1a contributed to both enhanced neuronal survival and neurite overgrowth in vitro [4]. Increased dendritic complexities is a shared feature of several neurological disorders associated with cognitive defects and is a conserved feature of $T s c l^{\text {null }}$ neurons across brain regions [11-14]. Considering the correlation between hyperactive mTORC1 and increased HIF1a and dendritic complexities, we examined whether HIF1a contributes to dendrite overgrowth in developing $T s c 1^{\text {null }}$ neurons.

To examine this question, we used postnatal neurogenesis as a model system in which neonatal electroporation in the neurogenic subventricular zone (SVZ) allows reliable plasmid expression in newborn neurons undergoing maturation and synaptic integration in the olfactory bulb (OB) $[15 ; 16]$. Using this approach, we previously reported consistent increase in mTORC1 
activity and dendritic complexity in newborn OB neurons [6;12;17] and increased Hifla mRNA levels as well as HIF1a transcriptional activity [6]. We found that genetically decreasing and blocking HIF1a level and function, respectively, led to a reduction of $T s c I^{\text {null }}$ dendritic overgrowth. Finally, HIF1a overexpression in developing wild-type neurons led to increased dendritic complexity suggesting that alterations in dendritic patterning are expected in pathological conditions associated with increased HIF1a expression.

\section{Methods}

\section{Animals}

Research protocols were approved by the Yale University Institutional Animal Care and Use Committee. Experiments and methods were carried out in accordance with the approved guidelines. Experiments were performed on littermate $T s c 1^{\mathrm{fl} / \mathrm{wt}}-\mathrm{R} 26 \mathrm{R}^{\mathrm{tdT} T o m a t o}$ and $T s c l^{\mathrm{fl} / \mathrm{mut}}-$ $\mathrm{R} 26 \mathrm{R}^{\mathrm{tdT} T o m a t o}$ mice of either gender obtained by crossing the following 2 lines of transgenic mice: $T s c 1^{f l f l}$ (Jackson Laboratories) and $T s c 1^{\text {wt } m u t}$ (cryorecovered from NCI) that we had precrossed with R26R-Stop-tdTomato mice (R26R $\mathrm{R}^{\text {tdTomato }}$, Jackson Lab). The Tsc Iflff $^{\text {and }}$ $T s c l^{w t / m u t}$ mouse lines were originally generated by David J. Kwiatkowski (Brigham and Women's Hospital, Harvard Medical School, Cambridge, Massachusetts, USA). Mice were prescreened for successful electroporation prior to sacrificing by viewing with an epifluorescence microscope or a Kodak 4000 imager.

\section{Genotyping}

Tail or toe samples were taken and were subjected to DNA isolation, PCR amplification using previously published primers [18] and amplicons separated by standard electrophoresis methods.

\section{Vectors}


The vectors pCAG-HIF1a, PBS/pU6-HIF1s RNAi plasmid 1 (constitutively expressed shRNA under U6 promoter), pCAG-HIF1a K532R (dominant negative HIF1a), pCAG-GFP, and pCAGCre were purchased from Addgene (donated by Dr. C. Cepko). The control shRNA contained an empty sequence in the same vector backbone. The pCAG-tdTomato was generated in our lab as previously described [19]. The luciferase (Luc) reporter vector was pcDNA3-rLuc-PolioIRESfLuc (Renilla and firefly Luc, rLuc, and fLuc) obtained from Dr. J. Blenis (Harvard) that was originally provided by Dr. Bitterman (University of Minnesota).

\section{Neonatal electroporation}

Postnatal electroporation was performed as previously described [15;20;21]. Plasmids (2-3 $\mu \mathrm{g} / \mu \mathrm{l})$ were diluted in PBS containing $0.1 \%$ fast green as a tracer. $0.5-1 \mu \mathrm{l}$ of plasmid solution was injected into the lateral ventricles of neonatal pups using a pulled glass pipette (diameter $<50$ $\mu \mathrm{m}) .5$ square-pulses of $50 \mathrm{~ms}$-duration with $950 \mathrm{~ms}$-intervals at $100 \mathrm{~V}$ were applied using the ECM 830 square wave pulse generator (BTX) and tweezer-type electrodes (model 520, BTX) placed on the heads of $\mathrm{P} 0-\mathrm{P} 1$ pups. The electrodes were positioned to direct current rostrally in the dorso-lateral SVZ.

\section{Luciferase assays}

Mice that had experienced postnatal electroporation were anesthetized with Nembutal and decapitated. OBs were flash frozen in liquid nitrogen and placed in $1.5 \mathrm{ml}$ ice cold lysis buffer and subjected to a dual luciferase assay (Promega) according to the manufacturer's recommendations. Tissue was passed through a pre-chilled 20 gage needle attached to a $1 \mathrm{ml}$ syringe 10 times. Samples were centrifuged at $10,000 \mathrm{~g}$ for $10 \mathrm{~min}$ at $4^{\circ} \mathrm{C}$. The supernatant was then placed on ice for $15 \mathrm{~min} .20 \mu \mathrm{l}$ of sample was preloaded into $100 \mu \mathrm{l}$ of luciferase assay substrate II and briefly mixed. The luminescence was measured in a TD 20/20 Luminometer 
(Turner Designs, Sunnyvale, CA, USA). Hundred microliters of stop and glo reagent were then added, mixed, and the luminescence from Renilla reniformis was determined.

\section{Morphometric analysis}

Postnatal day (P) 28 mice were deeply anesthetized with pentobarbital $(50 \mathrm{mg} / \mathrm{kg})$. The brain was then quickly removed and placed in $4 \%$ paraformaldehyde overnight at $4^{\circ} \mathrm{C}$, then washed in 1x PBS. The next day, 100- $\mu \mathrm{m}$-thick slices were prepared using a vibratome (Leica VTS 1000). Complete images of -tdTomato ${ }^{+}$neuronal basal dendrites were acquired in coronal sections using a Fluoview 1000 confocal microscope and 20X objective. Basal dendrites were traced using the 3D Z-stacks with simple neurite tracer software (FIJI, GNU GPL v3, an ImageJ plugin, URL: http://fiji.sc/Simple_Neurite_Tracer) from granule cells in the same layer across section and animals (deep layer). We chose to analyze basal dendrites for the following technical reason; the basal dendrite can be fully imaged and traced without confusion from a single labeled granule cell. Due to its length, the apical dendrite is often cut or partially cut, and this could lead to erroneous data. In addition, in the external plexiform layer where apical dendrites branch and terminate, it is difficult to distinguish whether the dendrite come from one cell or several cells. Sholl analyses were carried out using the number of intersections as a measure of morphological complexity. The number of intersections was measured in 5 or $10 \mu \mathrm{m}$-increment concentric circles and plotted as a function of the radial distance from the soma. Confocal Z-stacks from 3 different square fields of view were taken from each olfactory bulb (OB) section. This was done for 3 different $\mathrm{OB}$ sections in a randomly selected series from each animal. Analysis was performed blindly from at least 3 animals per condition.

\section{Statistics}


Analysis was performed on $\mathrm{N}$ indicating the number of animals in each condition or $\mathrm{n}$ indicating the number of cells/slices. Data were presented in Sigma Plot 11.0 or GraphPad Prism 6. Statistical significance was determined using unpaired Student t-test with $\mathrm{p}<0.05$ for significance

for all experiments except for Sholl analysis. For the later analysis, we used two way repeated measures (RM) ANOVA (with condition as a between groups factor and the number of crossings as a within group factor) with post hoc Bonferroni's test for comparison of dendritic crossing. Data are presented as mean \pm standard error of the mean (SEM).

\section{Results}

Tscl is one of two genes mutated in patients with TSC [22]. TSC1 (hamartin) forms a complex with TSC2 (tuberin) that negatively regulates mTOR through Rheb inhibition, thus Tsc1 loss results in increased mTORC1 activity (Fig. 1A). To delete $T s c 1$, we used $\mathrm{R}_{26} \mathrm{R}^{\mathrm{tdTomato}}$ mice containing a conditional (floxed, fl) and a wild type (wt) or Tscl mutant (mut) allele (noted $T s c I^{\mathrm{fl} / \mathrm{wt}}$ and $T s c I^{\mathrm{fl} / \mathrm{mut}}$ ) that mimics the genetics of TSC patients [14] and expressed a Cre recombinase (Cre)-encoding plasmid using neonatal electroporation. Neonatal electroporation was performed at postnatal day (P) 0 or 1 pups and allows plasmid expression into neural progenitor cells of the subventricular zone (SVZ) that generate neurons and glia with regional selectively [21;23-25] (Fig. 1B). Indeed, SVZ electroporation in neonates only leads to the labelling of newborn neurons in the OB and glia in the striatum and cortex. Rostral and dorsolateral electroporation preferentially leads to labeling of granule cells in the OB that display apical and basal dendrites but no axons [15]. Electroporation of Cre in the above Tscl mice allows Tscl excision and tdTomato expression in newborn granule cells of the OB [11]. In 
addition, we previously reported that $T s c 1$ deletion leads to increased mTORC1 activity, HIF1a levels and transcriptional activity, and dendritic complexity of granule cells [6;11].

Here, we confirmed that Cre electroporation in littermate $T s c l^{\mathrm{fl} / \mathrm{mut}}-\mathrm{R} 26 \mathrm{R}^{\mathrm{tdTomato}}$ and $T s c l^{\mathrm{f} / \mathrm{wt}}-\mathrm{R} 26 \mathrm{R}^{\mathrm{tdTomato}}$ led to increased dendritic complexity of $T s c l^{\text {null }}$ versus $T s c l^{\text {het }}$ neurons $(\mathrm{n}=34$ neurons each at $\mathrm{P} 28, \mathrm{~N}=4$ mice each, Figure 1C and D). Sholl-based dendritic analysis was performed on tdTomato+ granule neurons in the same layer of the OB (deep layer) and three sections per animal at the same rostral-caudal level across animals. We analyzed the basal dendrites for technical reason (see Methods). We examined whether decreasing HIF1a levels using short hairprin RNA would limit the dendritic overgrowth of $T s c 1^{\text {null }}$ neurons. $T s c 1^{\mathrm{fl} / \mathrm{mut}}$ mice were electroporated with Cre and a shRNA against HIF1a or a control shRNA (noted shHIF1a and shCTL, respectively). These knock down vectors were validated in our previous study using the same approaches [6]. The dendritic morphology of $T s c I^{\text {null }}$ neurons transfected with shHIF1a was compared to that of $T s c I^{\text {null }}$ and $T s c I^{\text {het }}$ neurons transfected with shCTL. Analysis was performed at P14 because the shRNA efficiency significantly decreases 2-3 weeks post-electroporation [17]. We found that HIF1a knockdown significantly reduced the dendritic complexity and total dendritic length of $T s c 1^{\text {null }}$ ( $\mathrm{n}>20$ neurons each, $\mathrm{N}=4$ each, P14, Figure 1C, D, and F). Because shRNA can have off-target effects, we used another approach to decrease HIF1a function; we co-electroporated a dominant negative HIF1a-encoding plasmid together with Cre and examined dendritic morphology at P14 as performed with the shRNA. As for the knockdown condition, we found that dnHIF1a decreased the abnormal dendritic complexity of $T s c 1^{\text {null }}$ neurons (Fig. 1E and F). 
Finally, we examined whether increases in HIF1a levels in wild-type neurons would lead to dendrite overgrowth. We used a HIF1a-encoding vector that significantly increased HIF1a protein expression when transfected in the mouse Neuro2a cell line (Fig. 2A). HIF1a regulates transcriptional activity of genes containing HIF response elements (HRE) within the promoter. We thus examined whether HIF1a-dependent gene transcription was increased following HIF1a plasmid electroporation using plasmids encoding constitutively active renilla luciferase or Photinus pyralis luciferase under the control of HRE. When the HIF1-encoding vector was coelectroporated with HRE and constitutively active luciferase gene reporters at P0, HIF1a overexpression produced a significant 4-fold increase in HRE transcriptional activation in P28 OB compared to OB containing GFP-expressing neurons ( $\mathrm{p}<0.001, \mathrm{~N}=11$ for HIF1a and $\mathrm{N}=6$ for GFP, Fig. 2B). For examining the effect of Hifla on dendritic complexity, wild type mice were electroporated with vectors encoding HIF1a and tdTomato or GFP and tdTomato (control). Sholl analysis was performed on the basal dendrites of P28 OB newborn neurons (Fig. 2C). HIF1a overexpression induced a significant increase in basal dendrite complexity and length $(\mathrm{n}=32$ and N=3 each, Fig. 2D and E). Thus, HIF1a overexpression in developing neurons is sufficient to induce transcriptional transactivation and increases dendritic complexity and length.

\section{Discussion}

Our novel finding is the contribution of HIF1a to abnormal dendritic patterning in $T s c 1^{\text {null }}$ neurons in vivo. These data are in agreement with previous in vitro studies showing that HIF1a contributes to dendrite overgrowth of cortical neurons following inflammation [4] as well as neurite outgrown in PC12 cells [26]. As shown in cortical neurons, we previously reported that HIF1a was important for the survival of newborn, olfactory bulb neurons in vivo [6]. Indeed, 
knocking down HIF1a levels led to the death of wild-type, $T s c 1$ heterozygote as well as $T s c 1^{\text {null }}$ neurons but to a lesser extent. Due to the massive loss of wild-type or Tscl heterozygote neurons, we could not explore the effect of knocking down HIF1a on the dendritic development of these neurons. Considering the cell loss following HIF1a knockdown, it is possible that the decreased dendritic overgrowth following HIF1a knockdown could result from poor health of $T s c l^{\text {null }}$ neurons. While it is difficult to dissociate death/survival from maturation and dendrite development, data showing that HIF1a overexpression increases the complexity and length of dendritic tree emphasize a function of HIF1a on dendritic development.

The question remains as to how HIF1a leads to dendritic overgrowth. It was previously reported that HIF1a controls neuronal survival and neurite overgrowth through regulation of NMDA receptor subunit NR1 expression [4]. In addition, we and others reported that NR1 controls the survival and dendritic development of developing neurons in the olfactory bulb [21;27]. It is thus possible that too much HIF1a leads to dendrite overgrowth by increasing NR1 expression, but NR1 expression has not been examined in TSC and this HIF1-NR1 needs to be further examined.

In conclusion, our study reports an intriguing function of too much HIF1a on dendrite development in both wild type and $T s c l^{\text {null }}$ neurons. Pharmacological inhibition of HIF1a activity is unfortunately not a good option for preventing dendritic overgrowth because it is lethal to neurons and in particular wild-type neurons [6]. Nevertheless, identifying the molecular mechanisms differentially controlling survival and dendrite growth could provide therapeutic options to limit dendritic overgrowth in TSC.

\section{Acknowledgements}


This work was supported by grants from the Department of Defense (Idea development award, W81XWH-10-1-0041), a National Institute of Health NRSA 10668225 and Epilepsy Foundation fellowship (D.M.F), and training grant from the China Scholarship Council (LZ). We thank the lab members for helpful discussion and comments.

\section{References}

[1] G.L.Semenza, Hypoxia-inducible factors in physiology and medicine, Cell 148 (2012) 399408.

[2] K.A.Zarember, H.L.Malech, HIF-1alpha: a master regulator of innate host defenses?, J. Clin. Invest 115 (2005) 1702-1704.

[3] F.R.Sharp, M.Bernaudin, HIF1 and oxygen sensing in the brain, Nat. Rev. Neurosci. 5 (2004) 437-448.

[4] S.H.Yeh, J.J.Hung, P.W.Gean, W.C.Chang, Hypoxia-inducible factor-1alpha protects cultured cortical neurons from lipopolysaccharide-induced cell death via regulation of NR1 expression, J. Neurosci. 28 (2008) 14259-14270.

[5] M.L.de Lemos, A.V.de la Torre, D.Petrov, S.Brox, J.Folch, M.Pallas, A.Lazarowski, C.BeasZarate, C.Auladell, A.Camins, Evaluation of hypoxia inducible factor expression in inflammatory and neurodegenerative brain models, Int. J. Biochem. Cell Biol. 45 (2013) 1377-1388.

[6] D.M.Feliciano, S.Zhang, J.L.Quon, A.Bordey, Hypoxia-inducible factor 1a is a Tsc1regulated survival factor in newborn neurons in tuberous sclerosis complex, Hum. Mol. Genet. 22 (2013) 1725-1734.

[7] N.El-Hashemite, V.Walker, H.Zhang, D.J.Kwiatkowski, Loss of Tsc1 or Tsc2 induces vascular endothelial growth factor production through mammalian target of rapamycin, Cancer Res. 63 (2003) 5173-5177.

[8] J.Brugarolas, W.G.Kaelin, Jr., Dysregulation of HIF and VEGF is a unifying feature of the familial hamartoma syndromes, Cancer Cell 6 (2004) 7-10.

[9] D.B.Shackelford, D.S.Vasquez, J.Corbeil, S.Wu, M.Leblanc, C.L.Wu, D.R.Vera, R.J.Shaw, mTOR and HIF-1alpha-mediated tumor metabolism in an LKB1 mouse model of PeutzJeghers syndrome, Proc. Natl. Acad. Sci. U. S. A 106 (2009) 11137-11142.

[10] J.F.Parkinson, B.Mitrovic, J.E.Merrill, The role of nitric oxide in multiple sclerosis., Journal of Molecular Medecine 75 (1997) 174-186.

[11] D.M.Feliciano, J.L.Quon, T.Su, M.M.Taylor, A.Bordey, Postnatal neurogenesis generates heterotopias, olfactory micronodules and cortical infiltration following single-cell Tsc1 deletion, Hum. Mol. Genet. 21 (2012) 799-810.

[12] C.A.Lafourcade, T.V.Lin, D.M.Feliciano, L.Zhang, L.S.Hsieh, A.Bordey, Rheb activation in subventricular zone progenitors leads to heterotopia, ectopic neuronal differentiation, and rapamycin-sensitive olfactory micronodules and dendrite hypertrophy of newborn neurons, J. Neurosci. 33 (2013) 2419-2431.

[13] L.Meikle, D.M.Talos, H.Onda, K.Pollizzi, A.Rotenberg, M.Sahin, F.E.Jensen, D.J.Kwiatkowski, A mouse model of tuberous sclerosis: neuronal loss of Tsc1 causes 
dysplastic and ectopic neurons, reduced myelination, seizure activity, and limited survival, J. Neurosci. 27 (2007) 5546-5558.

[14] D.M.Feliciano, N.W.Hartman, T.V.Lin, C.Bartley, C.Kubera, L.Hsieh, C.Lafourcade, R.A.O'Keefe, A.Bordey, A circuitry and biochemical basis for tuberous sclerosis symptoms: from epilepsy to neurocognitive deficits, Int. J. Dev. Neurosci. (2013) 667-678.

[15] B.Lacar, S.Z.Young, J.C.Platel, A.Bordey, Imaging and recording subventricular zone progenitor cells in live tissue of postnatal mice, Front Neurosci. 4 (2010).

[16] M.Pathania, L.D.Yan, A.Bordey, A symphony of signals conduct early and late stages of adult neurogenesis, Neuropharmacology 58 (2010) 865-876.

[17] L.Zhang, C.M.Bartley, X.Gong, L.S.Hsieh, T.V.Lin, D.M.Feliciano, A.Bordey, MEKERK1/2-Dependent FLNA Overexpression Promotes Abnormal Dendritic Patterning in Tuberous Sclerosis Independent of mTOR, Neuron 84 (2014) 78-91.

[18] D.M.Feliciano, T.Su, J.Lopez, J.C.Platel, A.Bordey, Single-cell Tsc1 knockout during corticogenesis generates tuber-like lesions and reduces seizure threshold in mice, J. Clin. Invest 121 (2011) 1596-1607.

[19] M.Pathania, J.Torres-Reveron, L.Yan, T.Kimura, T.V.Lin, V.Gordon, Z.Q.Teng, X.Zhao, T.A.Fulga, V.D.Van, A.Bordey, miR-132 enhances dendritic morphogenesis, spine density, synaptic integration, and survival of newborn olfactory bulb neurons, PLoS. ONE. 7 (2012) e38174.

[20] D.M.Feliciano, C.A.Lafourcade, A.Bordey, Neonatal subventricular zone electroporation, J. Vis. Exp. (2013).

[21] J.C.Platel, K.A.Dave, V.Gordon, B.Lacar, M.E.Rubio, A.Bordey, NMDA receptors activated by subventricular zone astrocytic glutamate are critical for neuroblast survival prior to entering a synaptic network, Neuron 65 (2010) 859-872.

[22] M.van Slegtenhorst, R.de Hoogt, C.Hermans, M.Nellist, B.Janssen, S.Verhoef, D.Lindhout, O.A.van den, D.Halley, J.Young, M.Burley, S.Jeremiah, K.Woodward, J.Nahmias, M.Fox, R.Ekong, J.Osborne, J.Wolfe, S.Povey, R.G.Snell, J.P.Cheadle, A.C.Jones, M.Tachataki, D.Ravine, J.R.Sampson, M.P.Reeve, P.Richardson, F.Wilmer, C.Munro, T.L.Hawkins, T.Sepp, J.B.Ali, S.Ward, A.J.Green, J.R.Yates, J.Kwiatkowska, E.P.Henske, M.P.Short, J.H.Haines, S.Jozwiak, D.J.Kwiatkowski, Identification of the tuberous sclerosis gene TSC1 on chromosome 9q34, Science 277 (1997) 805-808.

[23] A.T.Chesler, C.E.Le Pichon, J.H.Brann, R.C.Araneda, D.J.Zou, S.Firestein, Selective gene expression by postnatal electroporation during olfactory interneuron nurogenesis, PLoS. ONE. 3 (2008) e1517.

[24] C.Boutin, S.Diestel, A.Desoeuvre, M.C.Tiveron, H.Cremer, Efficient in vivo electroporation of the postnatal rodent forebrain, PLoS. ONE. 3 (2008) e1883.

[25] M.E.Fernandez, S.Croce, C.Boutin, H.Cremer, O.Raineteau, Targeted electroporation of defined lateral ventricular walls: a novel and rapid method to study fate specification during postnatal forebrain neurogenesis, Neural Dev. 6 (2011) 13.

[26] D.C.Genetos, W.K.Cheung, M.L.Decaris, J.K.Leach, Oxygen tension modulates neurite outgrowth in PC12 cells through a mechanism involving HIF and VEGF, J. Mol. Neurosci. 40 (2010) 360-366.

[27] C.W.Lin, S.Sim, A.Ainsworth, M.Okada, W.Kelsch, C.Lois, Genetically Increased CellIntrinsic Excitability Enhances Neuronal Integration into Adult Brain Circuits, Neuron 65 (2010) 32-39. 


\section{Figure legends}

Figure 1: HIF1a knockdown and genetic inhibition prevents dendritic overgrowth in newborn $\boldsymbol{T s c 1}^{\text {null }}$ neurons. (A) Simplified diagram illustrating the TSC1/TSC2-mTORC1-HIF1 pathway. (B) Diagram of a mouse brain receiving Cre plasmid electroporation at P0. By P14, newborn neurons have reached the olfactory bulb (OB). (C) Reconstructions of the basal dendrites of $T s c l^{\text {het }}$ transfected with a control shRNA (empty vector, shCTL) in $T s c 1^{\mathrm{fl} / \mathrm{wt}}$ mice and $T s c 1^{\text {null }}$ neurons transfected with either shCTL or shHIF1a in $T s c l^{\mathrm{f} / \mathrm{mut}}$ mice. (D) Sholl analysis for neurons whose examples are shown in (C). The statistical analysis (asteriks) refers to $T s c 1^{\text {null }}+\operatorname{shCTL}$ compared to $T s c l^{\text {null }}+\operatorname{shHIF1a}$. (E) Sholl analysis for $T s c l^{\text {null }}$ neurons coelectroporated with either GFP (control) or a HIF1a dominant negative vector (dnHIF1a). (F) Bar graphs of the total dendritic length of $\mathrm{OB}$ neurons inconditions shown under the bar. Statistical analysis of sholl data was performed with 2-way repeated measures ANOVA with post hoc Bonferroni's test. One way ANOVA was used for data in the bar graph. ***,p<0.0001, $* * *, \mathrm{P}<0.001$, and $\mathrm{s} * *: \mathrm{p}<0.01$.

Figure 2: Increased HIF1a expression leads to dendritic overgrowth in wild-type neurons.

(A) Western blot of HIF1 expression following transfected of Neuro2a cells 2 with a GFP or HIF1a overexpression plasmid. (B) Bar graphs of the relative luciferase activity induced by HIFfa overexpression in OB from P28 $T s c 1^{\text {wt/mut }}$ mice. (C) Representative examples of traced basal dendrites from GFP- or HIF1a-overexpressing granule cells. (D) Number (\#) of crossings as a function of the distance from control (open circles) and HIFla-overexpressing (black circles) granule cells. (E) Bar graphs of the basal total dendritic length in control and HIF1aoverexpressing neurons. Statistical analysis of sholl data was performed with 2-way repeated 
measures ANOVA with post hoc Bonferroni's test. Student t-test was used for data in the bar graph. $*$ p $<0.05 ; * *: \mathrm{p}<0.01$ 
Figure 1, Feliciano
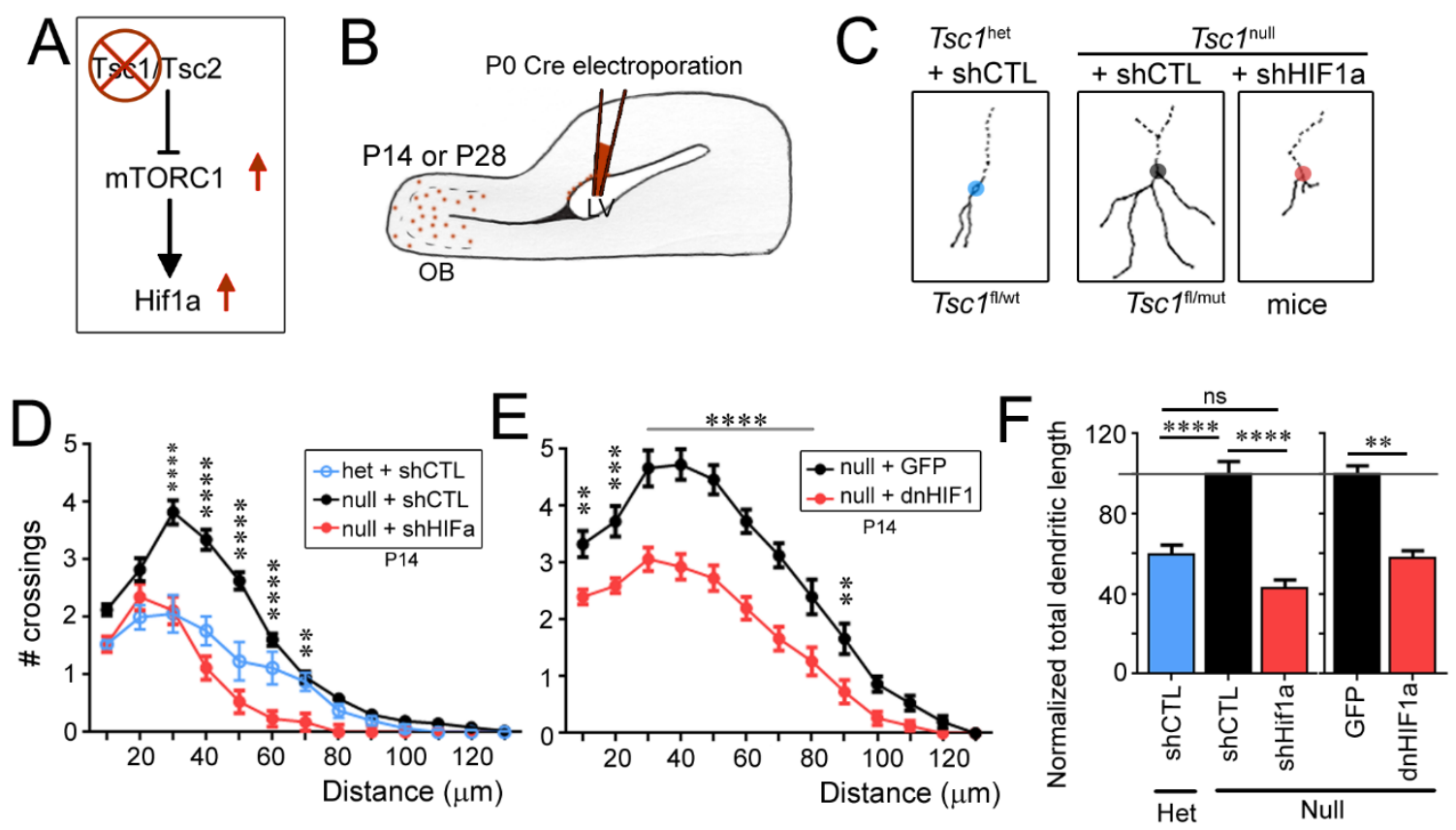
Figure 2, Feliciano
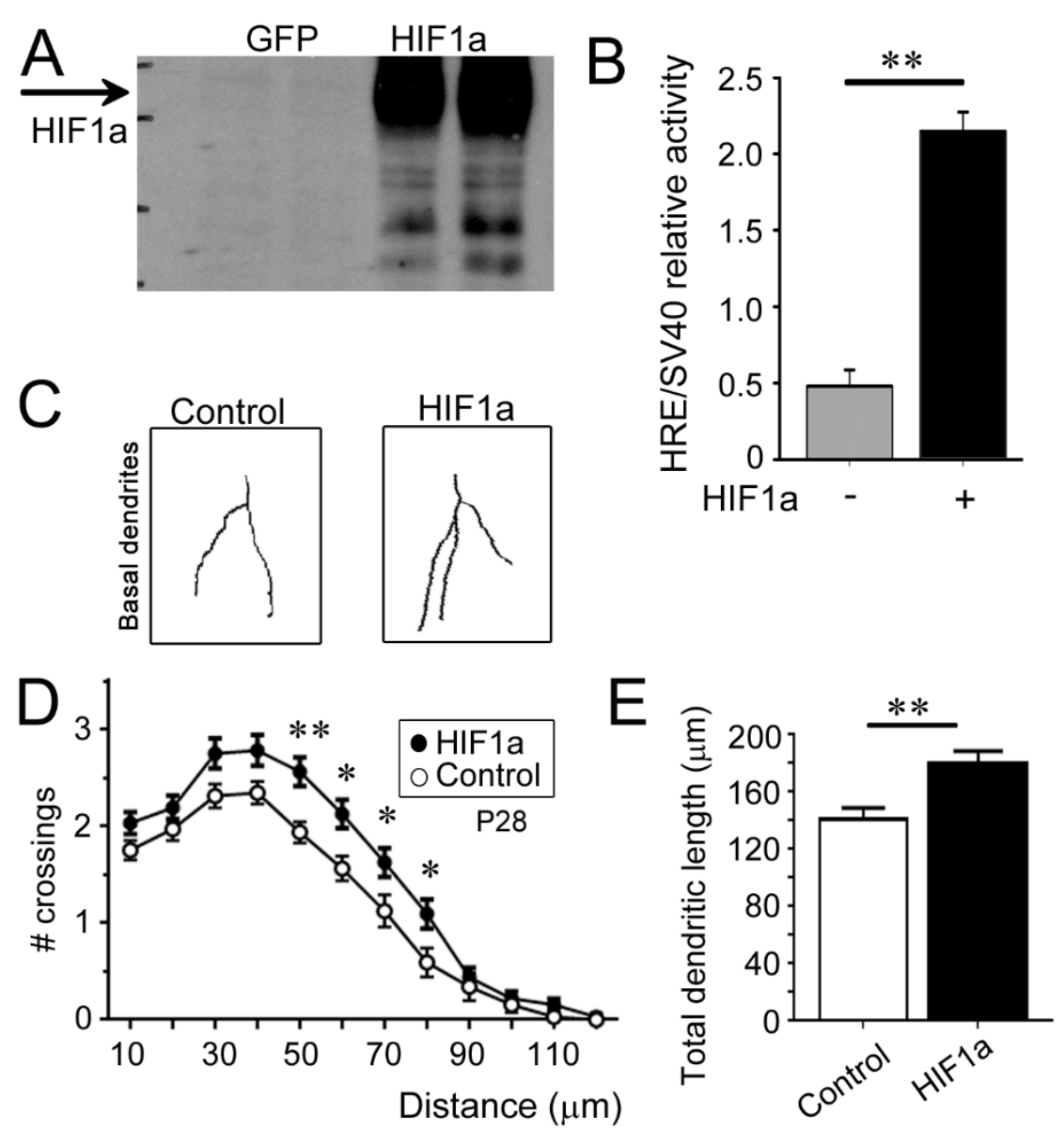